\title{
CMM MEASURing CYCle AND Human FACTOR
}

\author{
Martin Melichar, Dana Kubátová \& Jan Kutlwašer
}
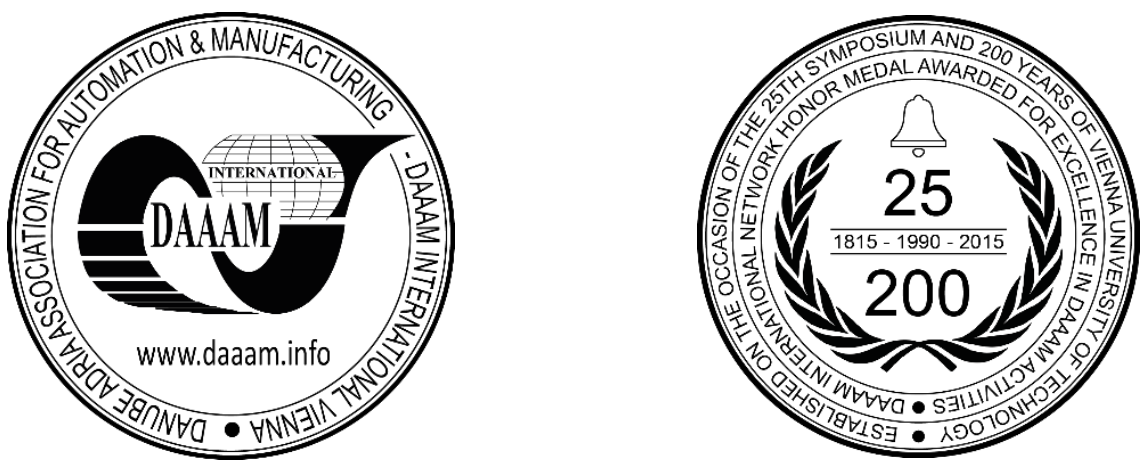

This Publication has to be referred as: Melichar, M[artin]; Kubatova, D[ana] \& Kutlwaser, J[an] (2016). CMM Measuring Cycle and Human Factor, Proceedings of the 27th DAAAM International Symposium, pp.0371-0376, B. Katalinic (Ed.), Published by DAAAM International, ISBN 978-3-902734-08-2, ISSN 1726-9679, Vienna, Austria DOI: $10.2507 / 27$ th.daaam.proceedings.055

\begin{abstract}
This paper deals with topic of evaluation of precise metrology application. Current state of measuring problems in the field od automotive industry often suppose that inspection of product guarantee the quality of this product. This idea is even stronger if the inspection is done by CMM. Research team tried to confirm or disprove importance of human factor for automatic measuring cycle of CMM. Using real parts with tolerances 11x bigger, that declared possibilities of CMM team of researchers done MSA analysis. Result of the experiment confirmed that the importance of human factor starts way above precision of CMM and it is necessary to focus on.
\end{abstract}

Keywords: MSA; CMM; measuring; metrology; measuring cycle.

\section{Introduction}

After being overlooked for a long time, metrology is gaining importance in today's world of technology, all the more so because of current standards in purchasing. In general, customer requirements do not end with products meeting their specifications, and with final inspection reports being presented as evidence of this conformity any more. An ever more frequent requirement concerns evaluation of supplier's measurement system to demonstrate its readiness, repeatability, reproducibility and suitability for the application in question. Final control of product is often implemented on a CMM. Here the question arises - how it affects the human factor in automatic mode CMM

\section{Theory of MSA}

There is a well-proven and customer-recognized methodology for demonstrating the qualification of a measurement system for a particular application: the MSA (Measurement System Analysis) quality tool. It uses simple experiments to find whether a particular approach chosen by the inspection personnel for a particular issue is suitable or at least adequate. One typical example involves verification of the required repeatability and reproducibility of results on pliable products, such as plastics, obtained by inspection personnel with a manual gauge that exhibits first-order error.

The outcome of the MSA analysis is the GR\&R parameter. [3] In practice, it is the percentage of the initial tolerance specified by the customer which the supplier "uses up" owing to ambiguous measurement. If GR\&R exceeds $30 \%$, the 
measurement system becomes unjustifiably expensive for the supplier (as it drives up the costs of manufacture which therefore must operate within a much narrower tolerance band), and unacceptable for the customer. [7]
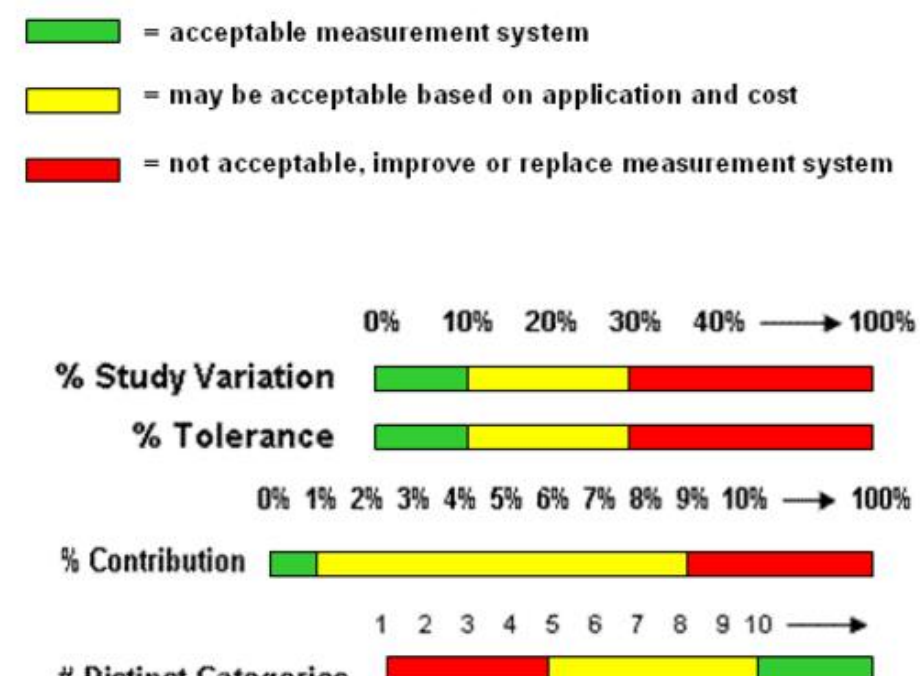

Fig. 1. MSA principle [2]

Manual measurement operations - to which MSA is traditionally applied - are subject to a number of factors linked to the human operator who possesses unique features including:

- Physical abilities

- Experience

- Attention

- Care

- Others.[6]

Since today's metrology laboratories are forced to rely on CMM systems, the effect of the operator can be expected to be minimized, provided that unified methodology and measurement programme are used. It order to test this hypothesis, the metrology laboratories of the Regional Technological Institute have conducted an experiment. [1]

\section{Experimental}

The measurement system analysis presented here involved CMM measurement of components from a single lot in large-series production. In the framework of MSA, the most widely used method was selected: assessment of average values and spread in a group of ten randomly chosen products from a single lot. The purpose of this experiment was to find whether MSA should be applied to the automatic mode of CMM operation as well or whether CMM can be relied on as a means of eliminating the operator influence. [4]

Input parameters of the experiment:

- On purpose, critical parts for automotive industry were selected whose tolerance was on the order of $0.01 \mathrm{~mm}$ (Fig.3)

- A suitable parameter - diameter of a hole - was chosen

- The measurement was performed on the Carl Zeiss Prismo 7 Navigator CMM (Fig.2) which itself guarantees high repeatability (approx. 1.5 micrometre using the index able head)

- For this measurement, a special program was developed for the part type

- All data collection took place under consistent controlled conditions $\mathrm{t}=20 \pm 1^{\circ} \mathrm{C}, 50 \%$ humidity

- Three operators each of whom possessed a different level of experience with CMM and who did not know each other were selected in order to avoid undesirable interference

- Using a measurement cycle, each operator measured 10 parts three times (in a random order) 


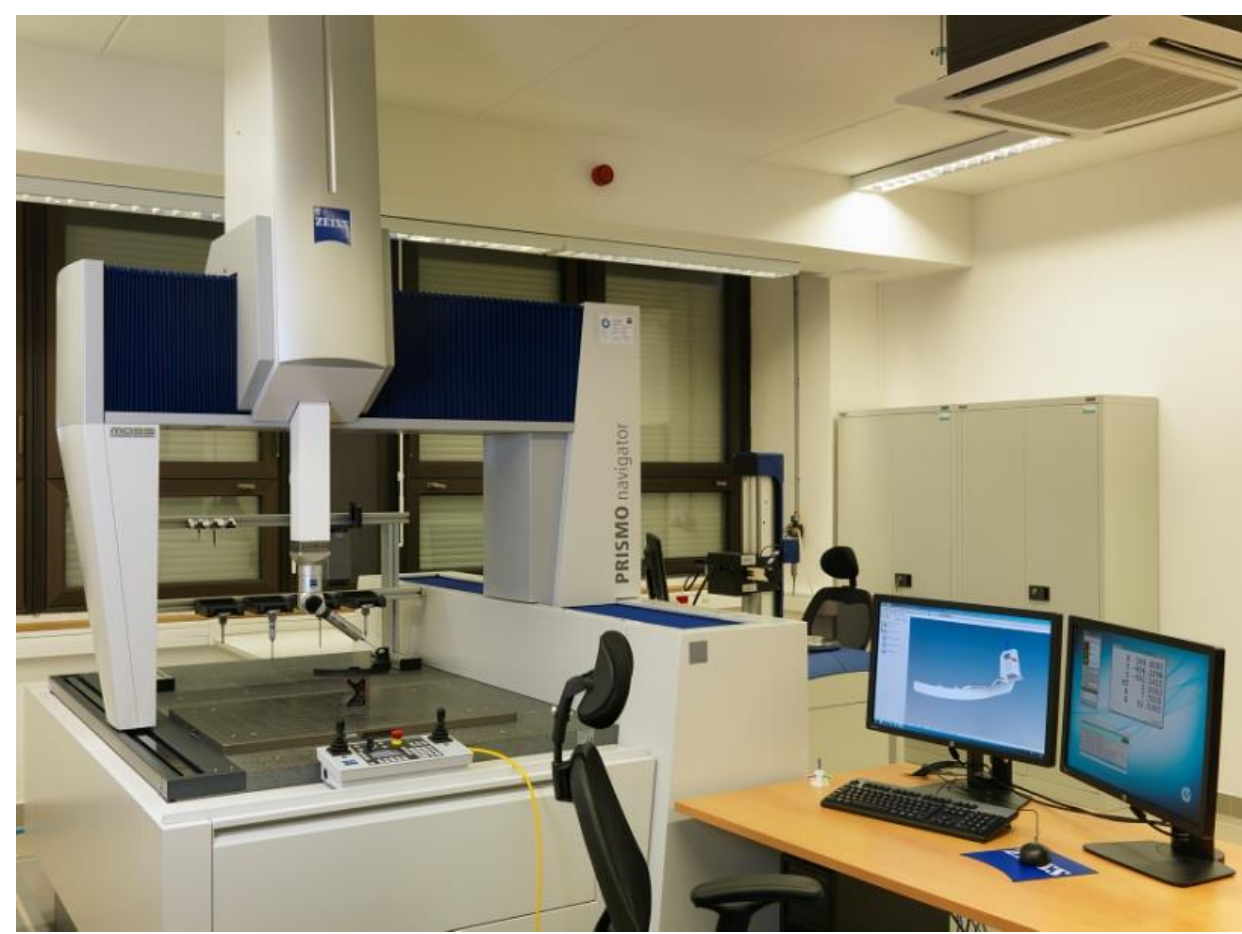

Fig. 2. Zeiss Prismo7 Navigator

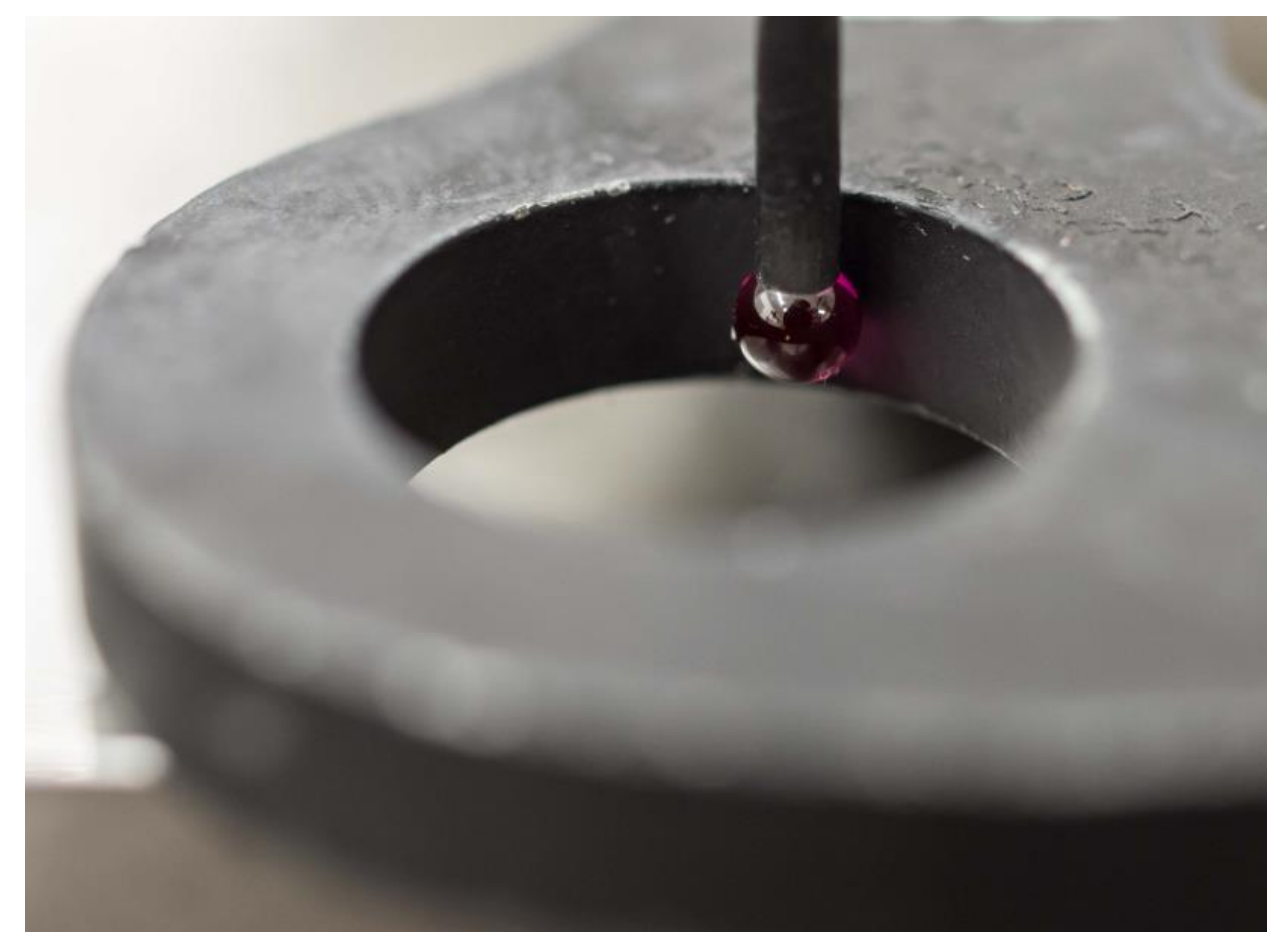

Fig. 3. Touch the measurement

The only theoretical potential complications affecting repeatability and repeatability are as follows:

- Machine's repeatability error

- Machine vibration - e.g. due to the speed of traversing mechanisms or due to the environment

- System points inadequately recorded by the operator

- Part inadequately clamped by the operator

- Incorrectly chosen stylus tip - e.g. its diameter

- Program error [5] 


\begin{tabular}{|c|c|c|c|c|c|c|c|c|c|c|c|}
\hline \multirow{2}{*}{\multicolumn{2}{|c|}{$\begin{array}{c}\text { Operator } \\
-- \\
\text { Measure- } \\
\text { ment series }\end{array}$}} & \multicolumn{10}{|c|}{ Part number } \\
\hline & & 1 & 2 & 3 & 4 & 5 & 6 & 7 & 8 & 9 & 10 \\
\hline \multirow{3}{*}{$\mathbf{A}$} & 1 & 15,430 & 15,428 & 15,428 & 15,437 & 15,426 & 15,433 & 15,438 & 15,436 & 15,438 & 15,428 \\
\hline & 2 & 15,428 & 15,428 & 15,429 & 15,437 & 15,431 & 15,432 & 15,439 & 15,436 & 15,437 & 15,428 \\
\hline & 3 & 15,430 & 15,425 & 15,428 & 15,435 & 15,426 & 15,432 & 15,440 & 15,436 & 15,436 & 15,427 \\
\hline \multicolumn{2}{|c|}{ Average } & 15,429 & 15,427 & 15,428 & 15,436 & 15,428 & 15,432 & 15,439 & 15,436 & 15,437 & 15,428 \\
\hline \multicolumn{2}{|c|}{ Spread } & 0,002 & 0,003 & 0,001 & 0,002 & 0,005 & 0,001 & 0,002 & 0,000 & 0,002 & 0,001 \\
\hline \multirow{3}{*}{ B } & 1 & 15,427 & 15,428 & 15,427 & 15,437 & 15,430 & 15,434 & 15,441 & 15,435 & 15,436 & 15,428 \\
\hline & 2 & 15,429 & 15,427 & 15,427 & 15,436 & 15,431 & 15,437 & 15,440 & 15,435 & 15,437 & 15,428 \\
\hline & 3 & 15,430 & 15,428 & 15,429 & 15,437 & 15,430 & 15,432 & 15,442 & 15,435 & 15,435 & 15,429 \\
\hline \multicolumn{2}{|c|}{ Average } & 15,429 & 15,428 & 15,428 & 15,437 & 15,430 & 15,434 & 15,441 & 15,435 & 15,436 & 15,428 \\
\hline \multicolumn{2}{|c|}{ Spread } & 0,003 & 0,001 & 0,002 & 0,001 & 0,001 & 0,005 & 0,002 & 0,000 & 0,002 & 0,001 \\
\hline \multirow{3}{*}{ C } & 1 & 15,430 & 15,429 & 15,430 & 15,436 & 15,430 & 15,434 & 15,440 & 15,435 & 15,438 & 15,427 \\
\hline & 2 & 15,426 & 15,426 & 15,429 & 15,438 & 15,429 & 15,434 & 15,440 & 15,437 & 15,438 & 15,427 \\
\hline & 3 & 15,424 & 15,426 & 15,429 & 15,437 & 15,430 & 15,432 & 15,440 & 15,437 & 15,436 & 15,429 \\
\hline \multicolumn{2}{|c|}{ Average } & 15,427 & 15,427 & 15,429 & 15,437 & 15,430 & 15,433 & 15,440 & 15,436 & 15,437 & 15,428 \\
\hline \multicolumn{2}{|c|}{ Spread } & 0,006 & 0,003 & 0,001 & 0,002 & 0,001 & 0,002 & 0,000 & 0,002 & 0,002 & 0,002 \\
\hline
\end{tabular}

Table 1. Table of measured values for the three operators in 10 repetitions

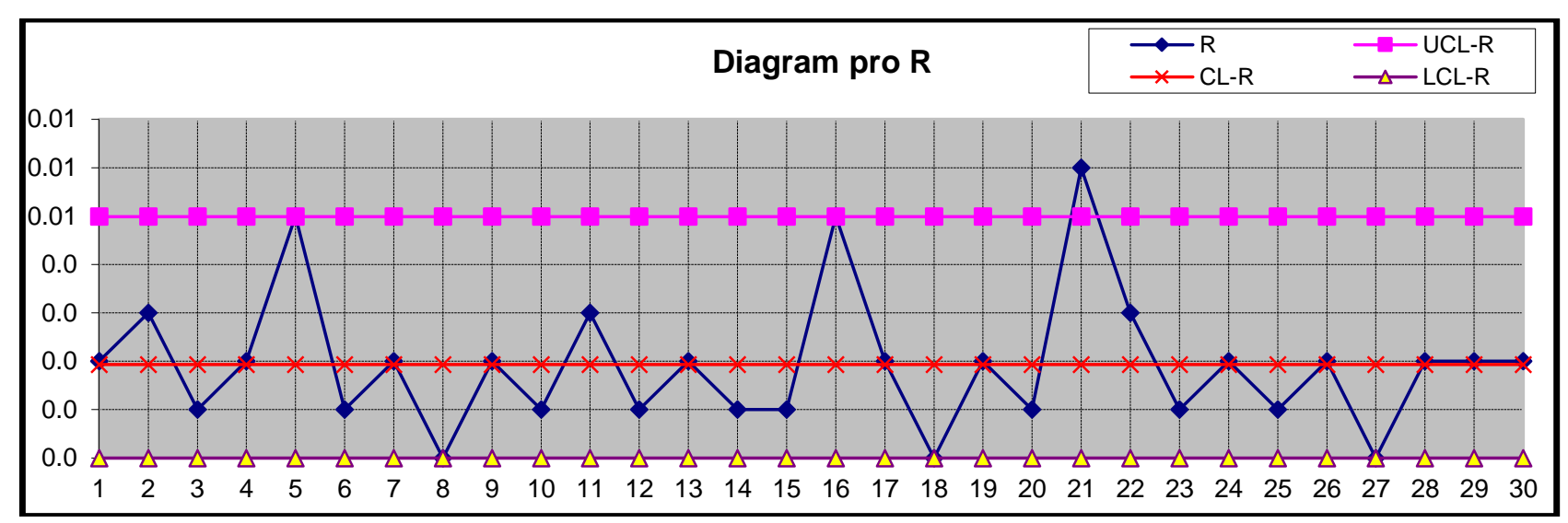

Fig. 1. Graph of scattering of the measured values 


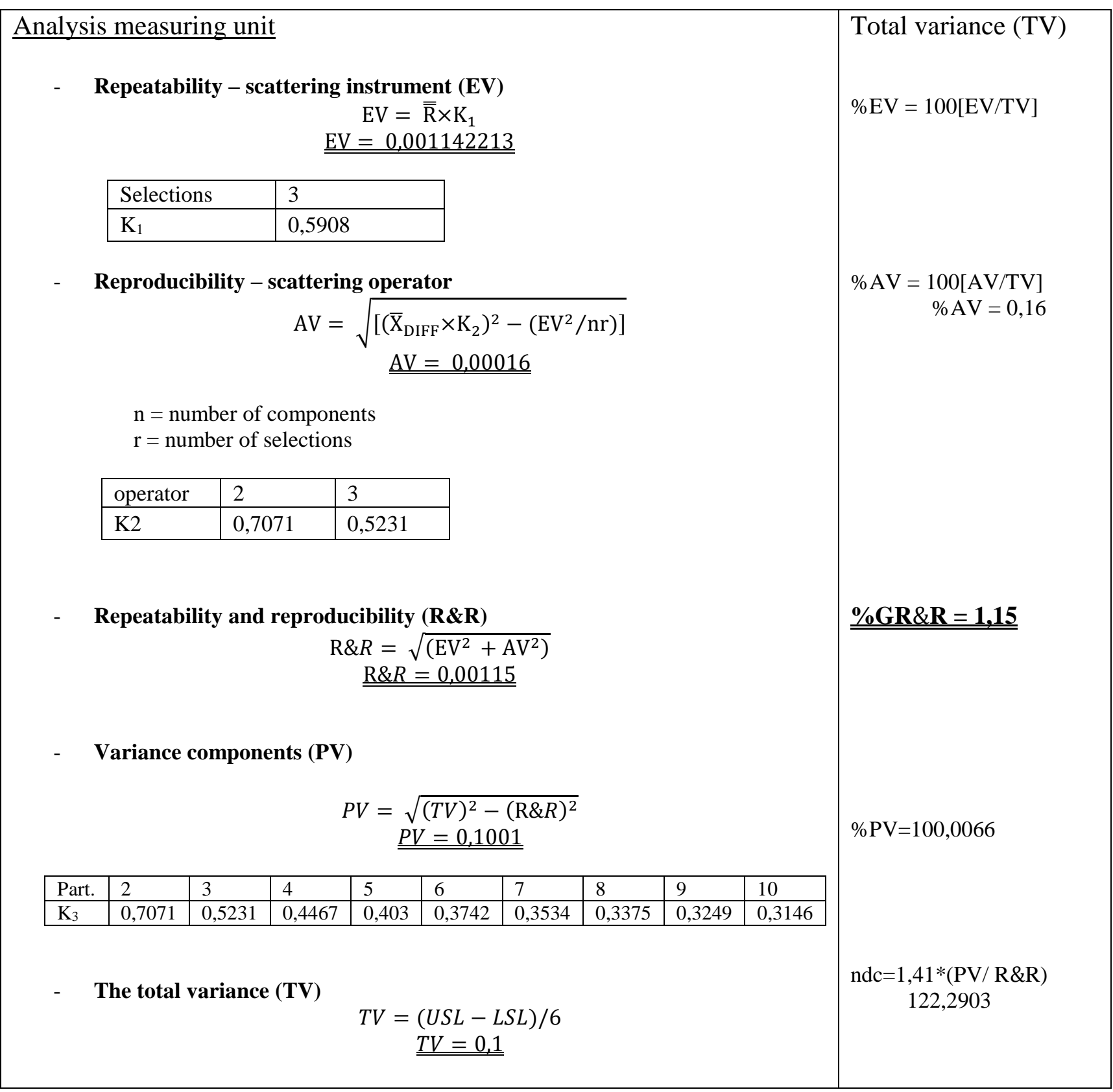

Table 2. Analysis measuring unit [7]

\section{Conclusion}

Using the experimental method was verified human factor influence on automatic CMM measurements. Applied methodology followed three independent operators, who controlled 10 parts. The experiment suggests that although the GR\&R value was found to be deep below the limit of 10 percent. For standard MSA analysis it is the inadmissible system and our measurement seems to be okay, but the results must be analysed extensively.

For instance, if it became necessary to measure components with much better accuracy than in our experimental arrangement, our analysis of our system could soon encounter unacceptable boundary values. Once the required component accuracy drops to approximately $0.005 \mathrm{~mm}$, the entire measurement process would have to be automated to such extent that at least the component clamping process and the initial identification of system points would have to be independent of human intervention.

There is one more important fact that must be taken into account - material component. A majority of those components where a request for MSA could be considered come from the field of processing of soft plastics where the potential effect of the operator can be even higher due to the force on the stylus. Inconsistencies speed of touch while operating by operator can be a significant source of uncertainty. 
It is therefore certain that monitoring repeatability and reproducibility of an automatic CMM in a supply chain of critical components is more than advisable. It can help reveal weak spots of the process which can ultimately lead to cost savings in production because a smaller portion of the available tolerance will be used up by measurement.

The investigation of effects of individual factors on the overall outcome of measurement is part of the project no. LO1502 B16.

\section{Acknowledgments}

This paper was supported by the project SGS-2016-005.

\section{References}

[1] Antonio Piratelli-Filho, Benedito Di Giacomo, CMM uncertainty analysis with factorial design, Precision Engineering, Volume 27, Issue 3, July 2003, Pages 283-288, ISSN 0141-6359, http://dx.doi.org/10.1016/S01416359(03)00035-7

[2] http://www.six-sigma-material.com/MSA.html [online]. [cit. 2016-09-08]

[3] Https://www.moresteam.com/toolbox/measurement-system-analysis.cfm: Measurement System Analysis (MSA) [online]. [cit. 2016-09-08]

[4] Matthias Asplund, Jing Lin, Evaluating the capability of measuring system for measuring the profile of the wheels using GR \& amp; R, vol. 92, October 2016, page 19-27, ISSN 0263-2241, 2016.05.090

[5] Burdick, Borror, Montgomery: A review of methods for measurement systems capability analysis (2003) Journal of Quality Technology, 35 (4), pp. 342-354. Cited 133 times. https://www.scopus.com/inward/record.uri?eid=2s2.0-1442324285\&partnerID=40\&md5=b85b8c9799a460da650f855106a13f11

[6] Wheeler: Problems with gauge R\&R studies(1992) Annual Quality Congress Transactions, 46, pp. 179-185. https://www.scopus.com/inward/record.uri?eid=2-s2.0-0026987231\&partnerID=40\&md5=3cbac4ee 9a86a24e46f38da58903721c

[7] http://www.rubymetrology.com/add_help_doc/MSA_Reference_Manual_4th_Edition.pdf [online]. [cit. 2016-10-06]

[8] Rewilak, J. MSA Planning - A proposition of a method (2015) Key Engineering Materials, 637, pp. 45-56. https://www.scopus.com/inward/record.uri?eid=2-s2.084924777858\&partnerID=40\&md5=a60d05220800743a43ac119db4560745 